\title{
A Multiple-Technique Approach to Inferring Bio-electrochemical Reaction Parameters
}

\author{
Henry O. Lloyd-Laney, ${ }^{\dagger}$ Nick D. J. Yates, ${ }^{\ddagger}$ Martin J. Robinson, ${ }^{\dagger}$ Alice R. \\ Hewson, ${ }^{\ddagger}$ Alison Parkin, ${ }^{*, \ddagger}$ and David J. Gavaghan ${ }^{*, \dagger}$ \\ $\dagger$ Department of Computer Science, University of Oxford, Wolfson Building, Parks Road, \\ Oxford, OX1 3QD United Kingdom \\ $\ddagger$ Department of Chemistry, University of York, Heslington, York, YO10 5DD, United \\ Kingdom \\ E-mail: alison.parkin@york.ac.uk; david.gavaghan@dtc.ox.ac.uk
}

\section{Abstract}

Uncovering the secrets of the biological Faradaic reactions, essential to the understanding of complex metalloenzymes, requires an information recovery process that is robust, rapid and replicable. This paper is a description of the workflow we have developed over the course of inferring chemical reaction parameters for a simple protein system, a bacterial cytochrome domain from Cellvibrio japonicus. This was a challenging task, as the signal-to-noise ratio in such protein-film voltammetry experiments is significantly lowered relative to the voltammetric data generated by simple chemicals. We have overcome these challenges by using a multiple-technique approach, which compensates for the difficulties inherent to analysis of the individual voltammetry experiments. We have shown that the parameters inferred are robust across multiple experiments performed for different preperations of the protein. This is an important proof-of-concept result for analysis of more complex metalloenzymes, which incorporate catalytic processes and multiple internal electron-transfer sites.

\section{Introduction}

Of the myriad chemistries that are required for the existence of life, some of the most truly fundamental such as splitting water, fixing nitrogen and transporting oxygen are underpinned by redox reactions. To understand these processes, which are achieved at standard temperatures and pressures using common metals and are consequently of significant biotechnological interest, $\stackrel{112}{2}$ we turn to protein-film voltammetry. In voltammetry, we use a time-varying potential to drive redox reactions, such that the recorded experimental current directly reports on the electron-transfer reactions that take place. In voltammetric analysis we attempt to reconstruct the underpinning reaction model that generated the experimentally measured current response. For a metalloprotein, the chemical reaction properties observed are controlled by the 3D-structure, which is defined by the protein sequence. Therefore the ultimate aim of our voltammetric approach is to understand how a protein relates sequence to its biological chemistry. In terms of the specific biological insights voltammetric analysis can offer, it allows quantification of the thermodynamics (the driving force) and the kinetics (the speed) of a reaction. By observing how those values change with different conditions, such as temperature, $\mathrm{pH}$ and the presence of inhibitors 
or substrates, we can build up a full mechanistic picture of biological redox reactions.

The choice of potential input has a very strong impact on the information we can recover from the resulting experimental current. The three voltammetry techniques used in this study are: purely sinudoidal voltammetry (PSV), as introduced in a previous piece of work, $\stackrel{3}{,}$ Ramped Fourier Transform Alternating Current Voltammetry (r-FTACV) as introduced by Bond and colleagues, 4 and Direct Current Voltammetry (otherwise known as Cyclic Voltammetry), or DCV, which has wide usage across the electrochemistry community. The three techniques are distinguished by their potential input. DCV uses a linear ramp, which can increase from a starting potential $\left(E_{\text {start }}\right)$ up to the switching potential $E_{\text {reverse }}$, and back down to $E_{\text {start }}$ or vice versa. The r-FTACV method uses a large-amplitude sinusoid overlaid on top of the DCV linear ramp, and PSV only uses the largeamplitude sinusoid. The large-amplitude sinusoid present in the potential inputs of r-FTACV and PSV results in a non-linear current response if an electroactive species is present in the system. This non-linearity can be observed in the Fourier transform of the current, where harmonic current responses (referred to hereafter as "harmonics") are observed at integer multiples of the frequency of the input sinusoid. Because the background current does not have the same degree of non-linearity (as evidenced by observing the Fourier transform of current from an unmodified electrode), we can consequently obtain a filtered signal that is hypothetically free of background. This is achieved by discarding the lower harmonics, resulting in a drastic improvement in signalto-noise. ${ }^{45}$ DCV does not elicit this non-linear response, and this makes separating the signal from the background highly challenging. ${ }^{6}$ The technique is still valuable to include firstly because of the widespread usage of DCV amongst the electrochemistry community, and secondly because DCV analysis for the single-electron case is relatively quick and simple, and can therefore provide a useful point of comparison for the results inferred from the other two tech- niques. Other techniques, particularly Square Wave Voltammetry (SWV) and Electrochemical Impedance Spectroscopy (EIS) have been used to interrogate biological systems, and in future work we hope to expand the framework we propose here to include these techniques.

Typically, the parameters that govern the behaviour of an electrochemical system, listed in table 1, cannot be measured directly. Instead, a we use a mathematical model (detailed in the supplementary information) that predicts an observed experimental measurement, usually the current, given defined values for the parameters. This act of simulating the current for a particular vector of parameters, $\theta$, is referred to as solving the forwards problem. For experimental data that fulfils the assumptions of our model, we assume that there will be a set of parameters that are representative of the actual chemistry; finding that parameter set is referred to as solving the inverse problem, or "parameter inference". There are multiple routes to solving the inverse problem in electrochemistry, reviewed ably elsewhere, and in this work we use two approaches minimisation, using the CMAES algorithm, which returns a best-fit parameter vector, and sampling, using a Markov-chain Monte Carlo approach, which yields a posterior parameter distribution, which can be analysed to determine the degree of correlation between the inferred parameter distributions. If the aim of understanding protein bio-electrochemistry by tracking changes in inferred parameters is to be realised, then parameter inference needs to be rapid, accurate and reproducible. The framework that we describe in this paper fulfils these three criteria. We have previously published on the topic of using an integrated experimental approach (using PSV and r-FTACV) when inferring parameters for ferrocene, a simple chemical system with excellent signal-to-noise ratios. 3 The next step, which this work describes, is to expand the framework such that it is appropriate for parameter inference on a biological system. There is an inherent difference between the signal-to-noise of protein vs. small-molecule voltammetry; biological sys- 
tems produce smaller signals, and larger and more complex background currents. There is a great deal of literature (including some detailed review papers ${ }^{9[10}$ ) related to attempts to infer parameters from PFV data, all of which in some sense require fitting a model to some observations. The analyses can be separated by the presence or absence of catalytic activity. For systems with no catalytic activity (i.e. purely Faradaic), there is more of a variety of voltammetric techniques and concomitant methods to extract features. For DCV, the features include oxidative/reductive peak-separation (as shown in a trumpet plot), $11+14$ peak-width $\underline{6[15}$ and background-subtracted total current. $\underline{13 \mid 16}$ For SWV, various current features, such as peak positions and normalised heights, 17 along with analysis of the Fourier transform of the current $\frac{18}{18}$ have been analysed. For r-FTACV, harmonic amplitudes ${ }^{12119}$ and the total harmonics 5112120 have both been used. For the case where catalytic processes are present, the overwhelmingly predominant technique is DCV, with some theoretical analyses exploring $\mathrm{SWV}, 21$ and the principal mode of analysis is the total current, 22 sometimes interpreted as a "waveshape".223 A large amount of effort and debate is expended on model development, as there are challenges related to distinguishing between different catalytic mechanisms, 24 along with challenges related to dispersion 25

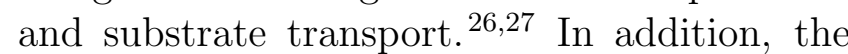
nature of the catalytic reactions means that boosting signal vs background by clever modulation of the input potential is not possible to the same extent as for Faradaic reactions, and naturally there is less innovation in this space. In addition, although the majority of the above efforts assume that the Faradaic reactions are well-described by Bulter-Volmer kinetics (as we do in this study), there are some efforts that incorporate Marcus theory instead. ${ }^{14}$ The variety of models, techniques and feature extraction processes referenced are indicative of the wide range of proteins that can be analysed using electrochemistry, as well as indicating something of an endless struggle to separate enough signal from background to be able to make valid inferences.
Our approach is a direct continuation of the work analysing biological Faradaic processes referenced above; what distinguishes it is the focus on the analysis of as much of the experimental current as feasible given the realities of background current, and the usage of multiple experiments to confirm inferred values. To showcase the utility of our approach, we have selected a simple protein from Cellvibrio japonicus, referred to as $\mathrm{CjX} 183$. C $\mathrm{jX} 183$ is a small domain of Cbp2D, a probable activating partner for the lytic polysaccharide monooxygenase protein active in $C$. japonicus ${ }^{28}$ LPMOs generally are known to improve the rate of cellulose digestion in a range of organisms, and are consequently of interest for biotechnological applications as they can facilitate biofuel production from biomass. $\frac{29}{29}$ The LPMO catalysed reaction requires reductive activation, and

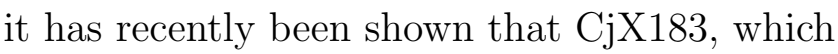
is a type-c cytochrome domain can carry out this LPMO activation. CjX183 was shown to undergo a single-electron reversible $\mathrm{Fe}^{3+/ 2+}$ Faradaic reaction when adsorbed on a carbon electrode, giving comparatively excellent signals in the process. $\frac{30}{30}$

The framework that we lay out in this paper is an attempt to leverage the advantages and mitigate the disadvantages of each voltammetry technique to provide a highly accurate protein redox analysis toolkit. On the leftmost plot in figure 1, we rank PSV and ramped experimental harmonics (obtained by inverse Fourier transforming the harmonic peaks discussed above), and the total current of a DCV experiment by their "interpretability" and their Faradaic information. We have shown the harmonics of the total current for the PSV and rFTACV cases as this is the form of the current that we actually analyse. The three plots on the right of the figure show actual experimental potential-time inputs (top row) and the resulting current (bottom row). In terms of Faradaic information, DCV is ranked last, because of the challenges associated with background subtraction. The highest ranked is r-FTACV, because it is highly sensitive to changes in Faradaic 

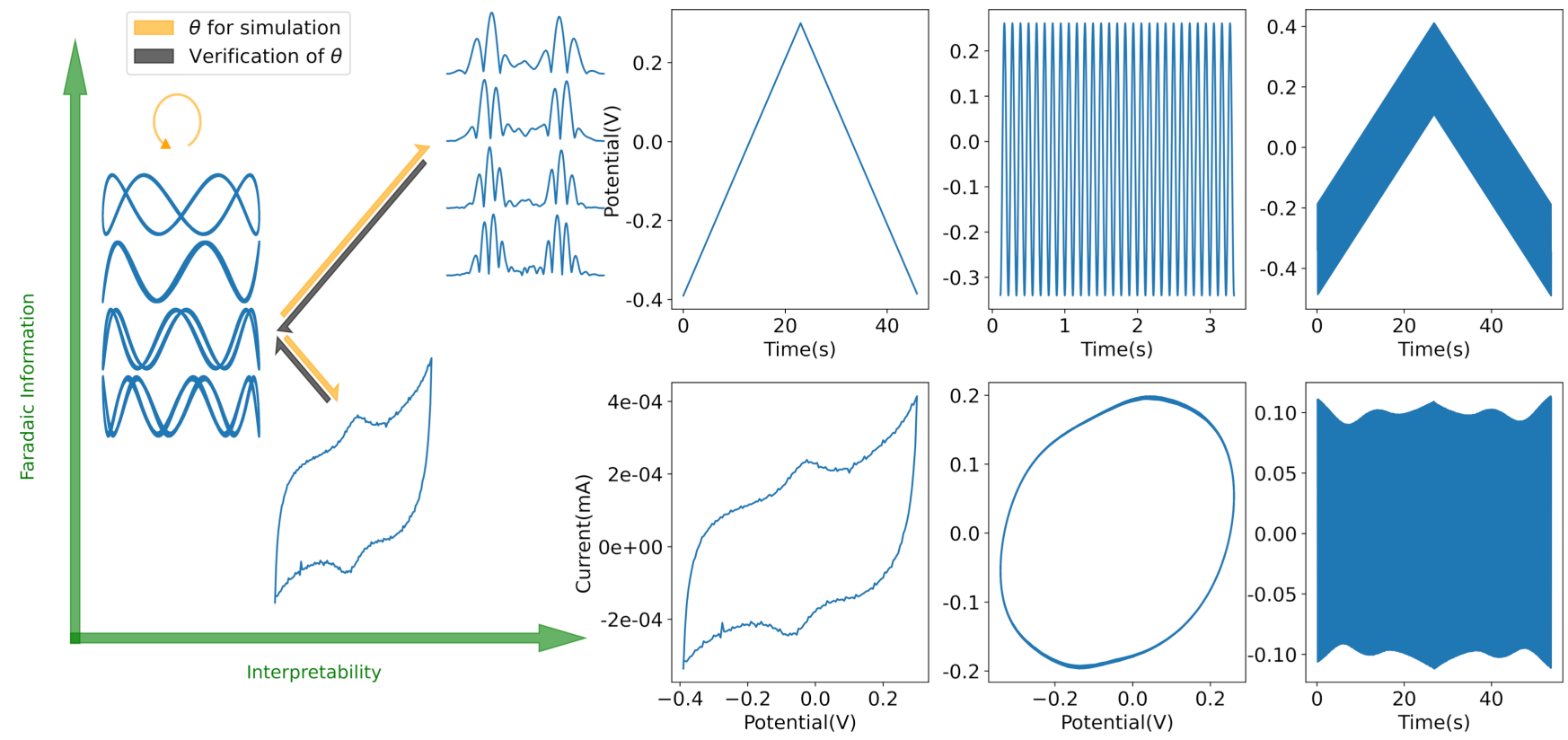

Figure 1: Left: The three experiments analysed in this paper, direct current voltammetry (DCV) as a current vs. potential plot, purely sinusoidal voltammetry (PSV) current harmonics 4-7 vs. potential, and ramped-Fourier Transform Alternating Current Voltammetry (r-FTACV) current harmonics 4-7 vs. time. The experiments are ranked according to how interpretable they are, and the amount of Faradaic information they provide. Orange arrows indicate simulation of current using a vector of chemical parameters $\theta$, and black arrows indicate that the results of these simulations are used to assess the goodness-of-fit. The other plots, from left to right, show the potential input (top row) and the current output (bottom row) for DCV, PSV and r-FTACV for real experimental data

parameters, an effect we explored in previous work $^{3}$ and the capacitive and Faradaic current contributions can be clearly separated by filtering. PSV sits between these two, and the reasons for this are laid out below. PSV is ranked last in terms of interpretability, because as we have shown,,$\frac{3}{3}$ the effect of the various reaction parameters on the appearance of the harmonics is less intuitive. Again, r-FTACV is ranked highest, as the effect of the distinct Faradaic parameters of interest can be determined through visual inspection. ${ }^{31} \mathrm{DCV}$ in this case is ranked between r-FTACV and PSV.

The obvious question after reading the above is, if r-FTACV is superior for all criteria, why use a technique other than r-FTACV? The answer is that r-FTACV is very expensive to simulate, being an order of magnitude slower than a 30-oscillation PSV or a DCV experi-

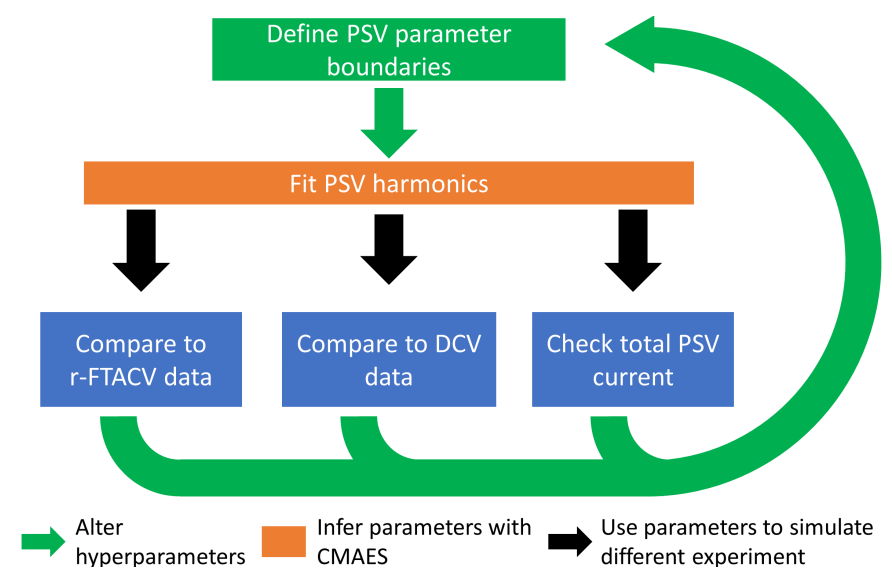

Figure 2: Flowchart showing the framework used to verify inferred bioelectrochemical reaction parameters 
ment. As solving the inverse problem takes a significant number of simulations (tens to hundreds of thousands), this seriously hampers any parameter inference effort. The effect is compounded for difficult problems, when there is uncertainty about the correct hyperparameters, such as how to bound parameter space, as this requires iterative parameter inference attempts for fine-tuning. Although we have used r-FTACV with some success for biological parameter inference, 20132 the timescales involved were not sustainable. Consequently, we have developed an inference process around PSV, to take advantage of the speed boost relative to r-FTACV. In previous work, $\stackrel{3}{b}$ we found that when inferring parameters from PSV currenttime data for a simple chemical system (ferrocene), there was one optimal parameter set which, when used to simulate a ramped experiment, also provided a good fit to r-FTACV data collected for the same electrode film. However, for the cytochrome data analysed in this paper, the signal-to-noise ratio is significantly worse, and we are forced to use filtering approaches to lessen the impact of background current, which was not necessary for the analysis of ferrocene. The challenge, as will be discussed below, is that this filtering discards a large quantity of Faradaic information. As a result, when fitting to filtered PSV data, we have found that we obtain multiple parameter vectors that generate a "good fit". This is exacerbated by the other weakness of PSV; because the filtered PSV current is largely uninterpretable, we cannot a priori select one parameter vector over another. To address this problem, we can use r-FTACV and DCV to verify which parameter vector is likely to represent the underlying chemical reality. The philosophy behind this verification approach is that, if all voltammetric experiments are conducted on the same electrode film, then the parameters inferred from one experiment should also describe data from another. We have represented this process graphically in figure 2. We infer parameters by fitting to the PSV harmonics, and then check their plausibility by predicting the current in a different form (such as the predicted DCV and r-FTACV currents, or the total PSV current), where we have experimental data for that form collected for the same film. As this data was collected from the same electrode film, a good fit in one experiment should predict a good fit in another. If this is not the case, then we determine which parameters might be responsible for a good fit for one set of experimental observations but not another, and then constrain parameter space to avoid this region. Examples of this process are laid out below; it requires knowledge about the chemically probable parameter ranges, as described in the Supplementary Information, in tables S1 and 2. In addition, this process requires experience with how the various parameters combine to give a particular waveshape, the better to understand how the effects of the various parameters combine to give a "good" or a "bad" fit. The cycle was repeated until we achieved the results shown in the rest of the paper. This process is a core justification for using PSV; if fitting to PSV data is ten times faster than fitting to r-FTACV data, we can go through multiple cycles of this process in the time required to undertake one r-FTACV inference attempt. As a consequence, this drives a more nuanced view about how the various parameters affect one another to provide a good fit, ultimately resulting in an approach that combines all three techniques to provide a robust approach to the overall parameter fitting process.

\section{Results and Discussion}

Examples of the the experimental current arising from DCV, PSV and r-FTACV voltammetric experiments interrogating surface-linked Cj183X are shown in figure 1. As can be seen, the signal in the DCV and r-FTACV experiments is significantly stronger than in the PSV experiment, where, to the eye, the signal (the two "bumps" at $\sim \pm 0.18 \mathrm{~V}$ ) is almost totally overwhelmed by background current. Consequently, we take advantage of the highly non-linear response of the Faradaic current, and filter the PSV current in the Fourier domain, artificially zeroing-out the region of the Fourier transform corresponding to harmonics 


\begin{tabular}{|c|c|}
\hline Symbol & escription \\
\hline$E^{0}$ & $\begin{array}{l}\text { The reversible potential - the potential at which the concentration of reduced and } \\
\text { oxidised species is equal }\end{array}$ \\
\hline$E^{0} \mu$ & The mean of a normal distribution of reversible potentials 31 \\
\hline$E^{0} \sigma$ & The standard deviation (s.d.) of a normal distribution of reversible potentials ${ }^{31}$ \\
\hline$k^{0}$ & The rate at which the redox reaction occurs \\
\hline$R_{u}$ & $\begin{array}{l}\text { The portion of solution resistance that is not compensated for by the potentiostat, } \\
\text { hence the "uncompensated resistance" }\end{array}$ \\
\hline$C_{d l}$ & $\begin{array}{l}\text { The magnitude of the background current arising from linear double-layer capaci- } \\
\text { tance effects }\end{array}$ \\
\hline$C_{d l E X}$ & $\begin{array}{l}\text { Terms used to model non-linear capacitance effects, where } \mathrm{X} \text { is the order number, } \\
\text { described in the mathematical model section of the supplementary information }\end{array}$ \\
\hline$\Gamma$ & The concentration of electroactive species on the surface of the electrode \\
\hline$\omega$ & The frequency of the input sinusoid (for PSV and r-FTACV) \\
\hline$\eta$ & The phase of the Faradaic current \\
\hline$C_{d l} \eta$ & The phase of the capacitive current \\
\hline
\end{tabular}

0-3, which were judged to have the largest capacitive contribution, from observation of PSV data from a blank electrode, as shown in the supplementary information in figure S1. This filtered Fourier transform is the form of the current that we use in solving the inverse problem, as opposed to the total current, which was what was used in. ${ }^{3}$ These harmonics are shown in figures 1, 3, 4 and 5, and represent our primary mode of analysis. The harmonics of the r-FTACV current are shown in figures 1, 3, 6 and 8 .

\section{Kinetic bounds}

In figure 3 we demonstrate how the PSV/rFTACV comparisons work in practice. A parameter vector generated by fitting to the 4th harmonic and above of the PSV current was used to generate both PSV and r-FTACV harmonics, which are plotted alongside the corresponding data harmonics in the left and righthand columns respectively. As described above, and shown in figure 2, an important part of obtaining a robust fit is constraining the parameter space in which the minimisation algorithm searches. We constrain parameter space firstly to prevent physically impossible parameter values, and secondly to exclude the areas of space that resulted in good fits to only the filtered portion of one experiment, and failed to generate a good-fit to current data from other techniques. The first parameter to be constrained for the latter reason was the kinetic parameter. This is because the kinetic parameter has a very clear impact on the appearance of r-FTACV harmonics, as discussed in previous work by our group 31 (and Alisters paper). Although the simulation harmonics plotted in figure 3 are a reasonably good fit to the data for the PSV case, the fit when translated to the r-FTACV harmonics is extremely poor, driven by a low inferred kinetic parameter. For r-FTACV, the higher the kinetic parameter, the closer in appearance the harmonics are to the "classical" [1 appearance, as shown in our previous work. ${ }^{5} \mathrm{As}$ the kinetic parameter decreases, the resolution of the individual harmonic lobes decreases, such that they start to merge with each other. By repeated r-FTACV/PSV comparisons of this type, we determined that the true kinetic parameter was at a minimum $50 \mathrm{~s}^{-1}$, and the lower bound for the optimisation algorithm was set accordingly. We also determined that the low

\footnotetext{
${ }^{1} \mathrm{~A}$ classical appearance is the one usually presented when introducing r-FTACV; 4 the harmonics are symmetrical and each lobe is clearly identifiable. This form is observed when the kinetic rate constants are highly reversible, when the capacitance and uncompensated resistance values are low.
} 


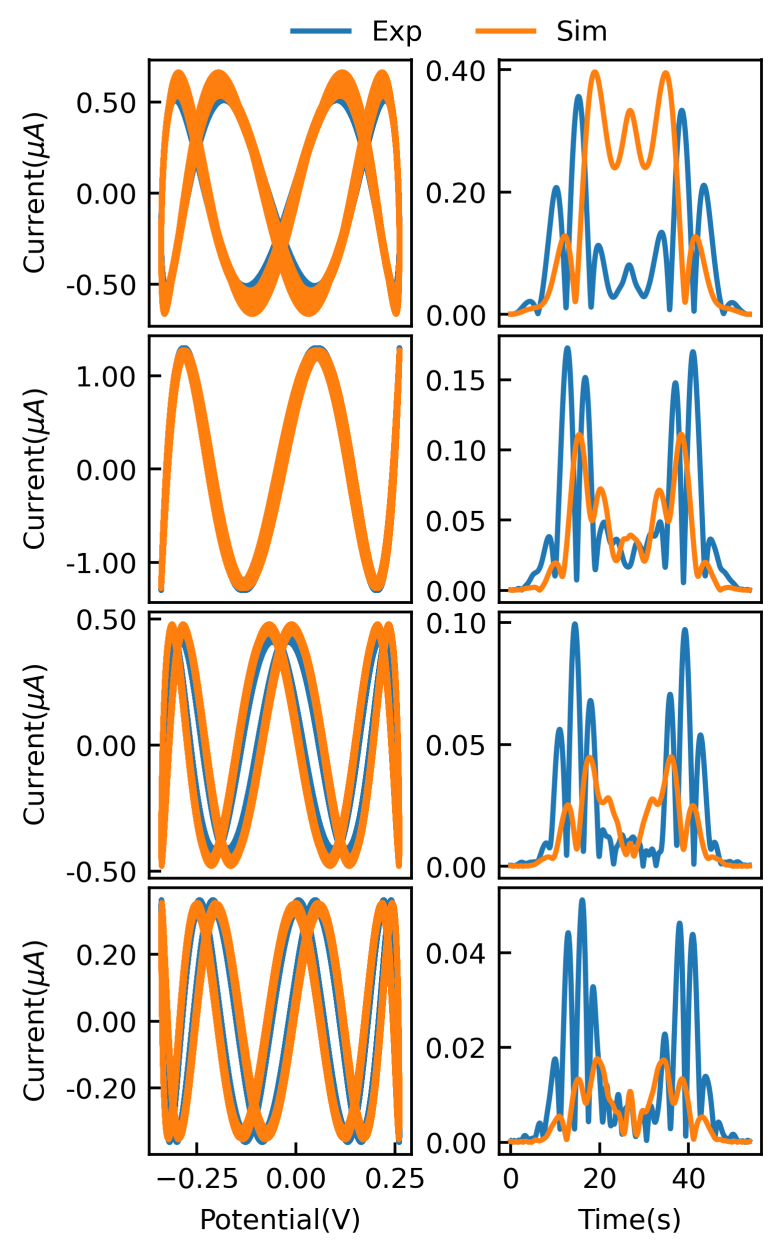

Figure 3: An example of how a relatively good agreement between PSV experimental (blue) and simulated (orange) harmonics 4-7 does not translate well to an r-FTACV harmonic comparison, driven by a low inferred $k_{0}$ value.

$k_{0}$ fits all returned phase and $C_{d l}$ phase values that were significantly different from the experimentally set value. Consequently, the phase and $C_{d l}$ phase bounds were set to $\pm 20 \%$ of the experimentally defined value of $\frac{3 \pi}{2}$

\section{Capacitance bounds}

The above bounding reduced the incidence of PSV parameter vectors that very obviously did not match the r-FTACV data. The next issue was as discussed above; although in previous work, we could infer a clear best solution vector that described both PSV and r-FTACV data, we have not been able to find such a solution for this dataset. Consequently, determining the difference between the "high $C_{d l}$ " and "low $C_{d l}$ " fits on the basis of the PSV and rFTACV harmonics alone, as shown in figure 4 was not possible. The two fits were primarily distinguished by large $(\sim 2$ orders of magnitude) difference in the inferred value of the linear double-layer capacitance value. This issue was resolved by plotting the predicted total PSV current - the high $C_{d l}$ parameter vector generates a total current 2-3x the magnitude of the experimental current, which is unrealistic. By the same token, it is not unrealistic that the total predicted current will be of a lower inferred magnitude than the total experimental current for the low $C_{d l}$ case. We do not expect the parameter inference approach to perfectly recover the true capacitance values, as by filtering the current we remove the majority of the capacitive contribution. However, for the high $C_{d l}$ case, the solver is artificially inflating the capacitance to achieve a better fit in the higher harmonics. This led to the realisation that, unfortunately, for a PSV experiment, high levels of capacitance can exert an effect on the higher harmonics (by suppressing their magnitude), a phenomenon that we believe is driven by spectral bleed from a very large first harmonic. Consequently it is important to guard against this effect by placing strict upper bounds (in this case, 1e-5F) on the value of the capacitance parameter. We did attempt to infer the true capacitance values for use in a "two-step" fitting approach as in previous work. ${ }^{32}$ However, we found that we could only obtain good fits to the filtered Fourier domain of the PSV current when fitting both Faradaic and capacitive parameters simultaneously. This illustrates the essential need for a multi-experiment methodology to allow the development of a robust approach to parameter inference.

\section{Inferring parameters from multi- ple films}

Using the bounds described above, we attempted to infer parameters for three sets of PSV cytochrome data, using three different electrode films, in order to gauge the reproducability of the fitting approach. The resulting best fits are shown in figure 5, with the inferred 

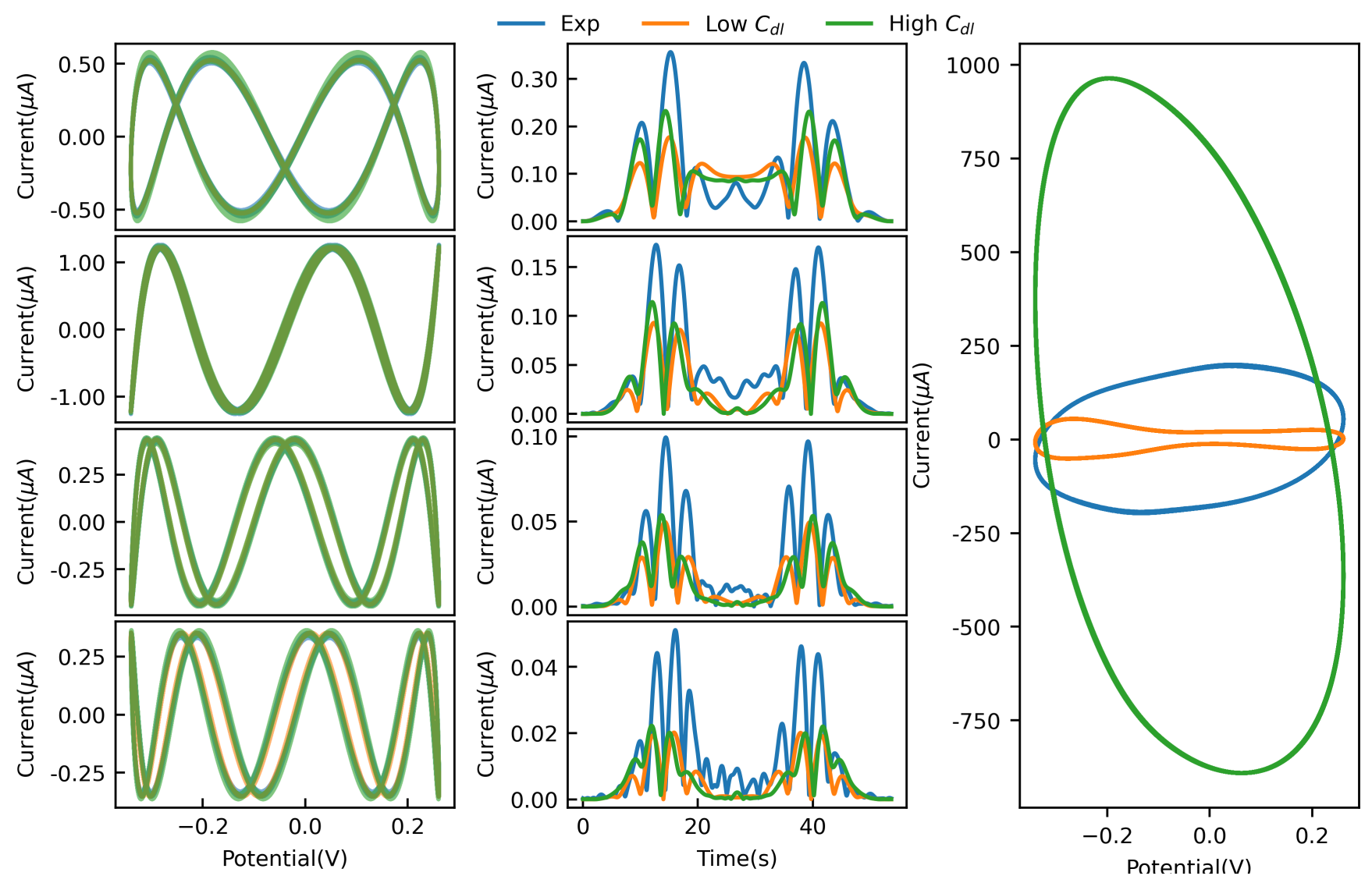

Figure 4: Comparison of two plausible parameter vectors generated by fitting to PSV harmonics 4 and above. In the leftmost plot, we see the very good agreement between simulated and experimental PSV harmonics 4-7 for both parameter vectors. Neither vector is clearly better than the other when compared against r-FTACV experimental harmonics in the central plot. The high $C_{d l}$ parameter was discounted on the evidence of the rightmost plot, as the predicted total current was significantly larger than the observed experimental current.

parameters shown in table 2, The bottom row of the figure are harmonics 4-10 for the experimental and simulated cases, plotted against the potential. The top row represents the region of the Fourier transform corresponding to harmonic 4 and above, inverse transformed back into current. In order to improve clarity, each of the 29 periods corresponding to a complete potential oscillation have been overlaid on top of each other, for the filtered PSV experiment and simulation. In addition, in order to show the amount of current we are discarding from our analysis, we have overlaid the total current on a different axis, in red. The total current has an amplitude approximately $8 x$ larger than that of the filtered current. However, we can observe why filtering is necessary; the Faradaic signal in the total current, occurring at approx- imately the maximum and minimum of the the red traces in figure 5, is almost totally obscured by the signal arising from capacitance, which makes up the rest of the plot.

In terms of the parameter values themselves, as reported in table 2, it is essential to note that in all cases the reported $\alpha$ value is the value of the upper bound, as is the value for the capacitance for PSV experiments 2 and 3. For $\alpha$, it was our experience that the solver converged to the value of the upper bound regardless of what it was set to. This is not unexpected - as detailed in our previous work, when the kinetic regime is reversible or quasi-reversible, the effect of the symmetry factor is low. We set the upper bound at 0.6 for reasons of chemical plausibility, but it is common practice to set 

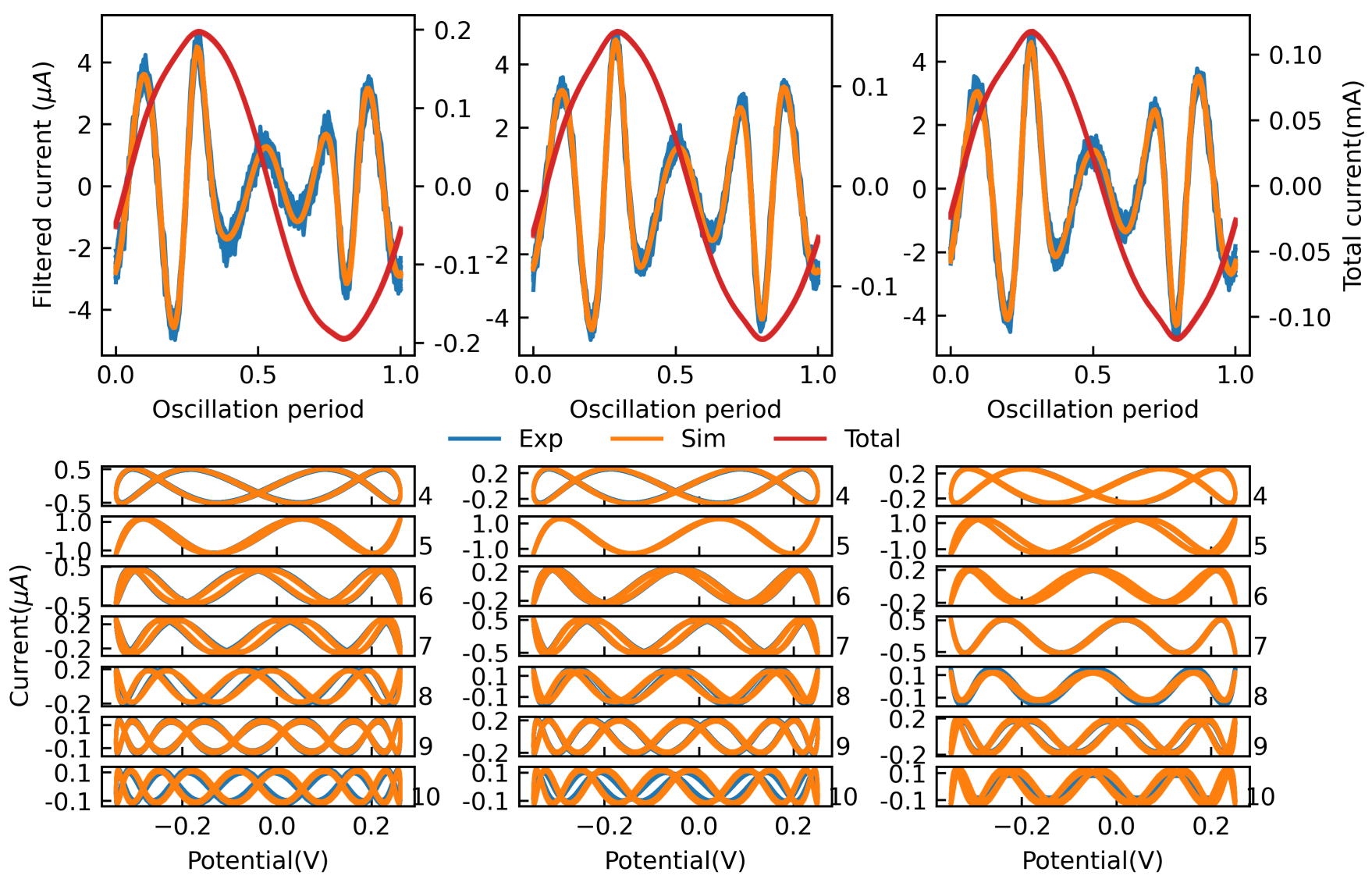

Figure 5: Simulated and experimental data for three separate electrode films. The simulation parameters for the three experiments (PSV 1, 2 and 3, from left to right) can be found in table 2 . The top row is a representation of PSV harmonic 4 and above, where the frequency domain, filtered such that harmonics 0-4 were set equal to 0, was inverse Fourier transformed, and then each of the current regions corresponding to a single potential oscillation was overlaid. This process was done for both the experimental and simulated timeseries. The total, unfiltered experimental current, is plotted on the same $x$-axis but a different $y$-axis, as it is of much greater magnitude. In the bottom row, PSV harmonics 4-10 were individually Fourier transformed and plotted against potential. 
Table 2: Best fit parameters for harmonics 4 and above of PSV experiments 1,2 and 3 . The resulting simulated PSV current is shown in figure 5. The same values were used to generate r-FTACV simulations shown in the top row of figure 6, and the values in brackets were used to generate the r-FTACV simulations in the bottom row of the figure

\begin{tabular}{|c|c|l|l|l|}
\hline Parameter & Symbol & PSV 1 & PSV 2 & PSV 3 \\
\hline \hline Midpoint potential mean & $E^{0} \mu(\mathrm{V})$ & $\begin{array}{l}-7.167 \mathrm{e}-2 \\
(-6.187 \mathrm{e}-2)\end{array}$ & $\begin{array}{l}-6.784 \mathrm{e}-2 \\
(-6.322 \mathrm{e}-2)\end{array}$ & $\begin{array}{l}-6.538 \mathrm{e}-2 \\
(-6.118 \mathrm{e}-2)\end{array}$ \\
\hline Midpoint potential standard deviation & $E^{0} \sigma(\mathrm{V})$ & $\begin{array}{l}0.045 \\
(0.033)\end{array}$ & $\begin{array}{l}0.053 \\
(0.036)\end{array}$ & $\begin{array}{l}0.051 \\
(0.035)\end{array}$ \\
\hline Rate constant & $k_{0}\left(s^{-1}\right)$ & 173.809 & 176.481 & 172.894 \\
\hline Uncompensated resistance & $\mathrm{R}_{u}(\Omega)$ & 148.688 & 316.812 & 81.525 \\
\hline Linear double-layer capacitance & $C_{d l}(\mathrm{~F})$ & $9.794 \mathrm{e}-6$ & $1.000 \mathrm{e}-5$ & $9.999 \mathrm{e}-6$ \\
\hline $1^{\text {st }}$ order $C_{d l}$ & $C_{d l E 1}$ & 0.014 & 0.079 & 0.095 \\
\hline $2^{\text {nd }}$ order $C_{d l}$ & $C_{d l E 2}$ & 0.04 & 0.021 & 0.045 \\
\hline $3^{r d}$ order $C_{d l}$ & $C_{d l E 3}$ & $-5.607 \mathrm{e}-4$ & $-4.381 \mathrm{e}-4$ & $-3.772 \mathrm{e}-4$ \\
\hline Surface coverage & $\Gamma\left(\mathrm{mol} \mathrm{cm}^{-2}\right)$ & $1.35 \mathrm{e}-11$ & $2.05 \mathrm{e}-11$ & $1.79 \mathrm{e}-11$ \\
& & $(1.68 \mathrm{e}-11)$ & $(1.83 \mathrm{e}-11)$ & $(1.45 \mathrm{e}-11)$ \\
\hline Potential frequency & $\omega(\mathrm{Hz})$ & 9.015 & 9.015 & 9.015 \\
& & $(8.96)$ & $(8.75)$ & $(8.83)$ \\
\hline $\mathrm{C}_{d l}$ phase & $\mathrm{C}_{d l}$ phase $(\mathrm{rads})$ & 4.729 & 4.696 & 4.712 \\
\hline Phase & Phase $(\mathrm{rads})$ & 4.572 & 4.599 & 4.628 \\
\hline Symmetry factor & $\alpha$ & 0.6 & 0.6 & 0.6 \\
\hline
\end{tabular}

the value of $\alpha$ to 0.5 under these conditions. When we attempted this, the inferred kinetic values were totally reversible (i.e. hit the upper kinetic boundary regardless of its value), which yielded worse fits when comparing the ramped harmonics (as we do for the parameters in table 2 in figure 6). In terms of the capacitance upper bound, for experiments 2 and 3 we found the solver converged to unrealistic capacitance values, such as plotted in figure 4. These effects are driven in part by parameter compensation; to compensate for not being able to increase or decrease a parameter beyond a certain limit, the solver will scale other, correlated parameters to achieve a similar effect. An illustration of which model parameters are correlated (excluding $C_{d l}$ and $\alpha$ ) is shown in figure 10 . For $\alpha$ and $C_{d l}$, the compensated parameter we are most interested in was the kinetic value. In the case of $\alpha$, a decrease of the upper $\alpha$ bound resulted in an increase in the kinetic parameter of $40 \mathrm{~s}^{-1}$ on average, before moving into totally reversible kinetic territory at an $\alpha$ value of 0.533 . For the capacitance, increasing the upper bound to 1e-4F, (an unrealistic value) caused highly divergent changes in the inferred kinetic value, with worse observed fits to both the PSV and ramped harmonics. What these results demonstrate is that, while we can fit well to the data, the exact values that we report are dependent on how parameter space is bounded. Consequently, it is important to describe and justify how this bounding is achieved, and to show exactly how we adjust parameters when attempting to compare results between experiments. The fact that we can fit three different experiments with parameters that are in the same regime implies that we are not merely in a local minima, and that the values we report are a reflection of the underlying chemistry. We note that for some parameters the value returned is the upper bound, which means that the choice of upper bound has affected the other reported parameter values, because of parameter compensation effects detailed above. We have determined a region of parameter space which gives consistently good fits to the PSV data, and the values we report are a sin- 
gle point within that parameter space. The precise values are dependent on our choice of certain bounds and could therefore be considered slightly arbitrary. However, we can make the claim that within this highly constrained region of parameter space lies the values that truly describe the underlying chemistry.

As mentioned above, we use r-FTACV data to act as a "check" on the values inferred by the PSV inference process. The result of this comparison for PSV experiments 1-3 is shown in figure 6. The top row uses the PSV simulation parameters written in table 2, except for the input frequency and phase. For the bottom row, we performed a limited optimisation on parameters thought likely to change as a result of so-called "film-loss" effects, in particular, the $E^{0} \mu, E^{0} \sigma$ and $\Gamma$ parameters. The altered values are written in brackets in table 2. We do this to show that only a relatively small modification in the PSV parameters is required to provide an excellent fit to the ramped harmonics further evidence that the PSV fit is representative of the chemical activity on the electrode. However, it should be noted that further investigation of the film-loss effect implied that the $E^{0} \sigma$ value predicted by the PSV parameters is too large (i.e. we do not observe a broadening of the harmonics to the extent shown in the top row of 6). This is partially driven by the imposition of capacitance bounds - increased $E^{0} \sigma$ is known to be "compensatory" for an artificially lowered $C_{d l}$ value. It should be noted that this takes us away from the philosophy of verification, because we are making alterations to the inferred parameters in order to make the translation between two experiments more convincing. However, we believe that this is an unavoidable consequence of the efforts required to remove the contribution of capacitance. The process of using PSV to obtain the approximate parameter regime, and then a single limited optimisation r-FTACV approach is still significantly faster than undertaking a de novo r-FTACV inference approach.

\section{The problem of DCV}

\section{Analysing a single experiment}

In the preceding sections, we have extensively discussed the interplay of PSV and r-FTACV. We now discuss how DCV data, obtained from the same electrode, can be used to validate the results above. DCV data was collected alongside PSV experiments 1 and 3. For PSV experiment 1 , the DCV data was only collected at one scan rate, and although the level of signal was extremely good for this type of experiment (as can be observed in the leftmost plot of figure 7), we found that DCV data was not particularly helpful when attempting to solve some of the issues with the inference process described above. The difficulties were multifaceted; the first issue was that our polynomial capacitance approximation could not accurately describe the capacitance current that occurred directly after the switching potential (from $0.25 \mathrm{~V}$ to $0.15 \mathrm{~V}$ in the DCV current in figure 7), partly because of the discontinuity in the potential input that occurs at the switching potential. The only way to achieve the "curved" appearance was with extremely high resistances (>1e $5 \Omega$ ), as in work by Feldberg, 33 which were incompatible with our PSV and r-FTACV fits, as well as with the observed Faradaic signal. The standard tool to get around this is using "background subtraction", as specified in previous work. ${ }^{6}$ We do not merely subtract purely capacitive current generated from an unmodified electrode, as the presence of a protein on the electrode also causes a change in the observed background current. The background subtraction methodology is shown by the plot on the left hand side of figure 7. The assumed background current (the third-order polynomials in red) is subtracted from the total current to get the background subtracted current in orange. In the middle plot, we show the same backgroundsubtracted current, and purely Faradaic current simulations. The DCV current parameters, listed in 3 , were obtained by fitting in the time-domain to the background-subtracted current, and the r-FTACV parameters are as detailed in table 2 for r-FTACV 1 . What is apparent is that there is no correspondence between 

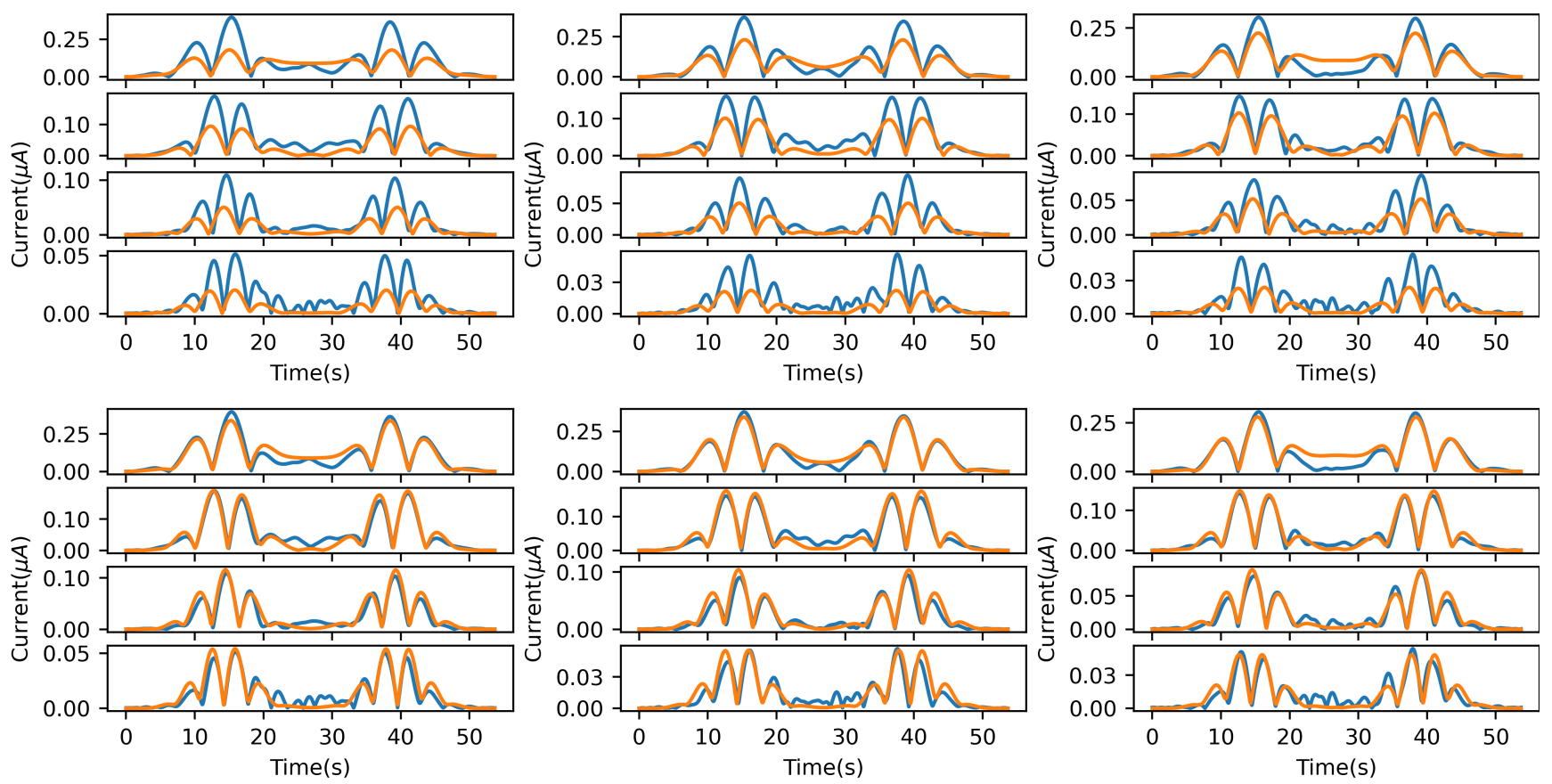

Figure 6: r-FTACV experiments conducted on the same electrode films as the corresponding PSV experiments shown in figure 5, plotted with simulations. In the top row, the simulations are generated using the PSV parameters in table 2. The lower row uses the same parameters apart from where there are values in brackets, which are used instead. For all three experiments, the input frequency for r-FTACV was slightly different for the one used in the PSV experiments. In the simulation, these were set to $8.96,8.75$ and $8.83 \mathrm{~Hz}$ for r-FTACV experiments 1,2 and 3 respectively. The values for phase and $C_{d l}$ phase were set to 0 

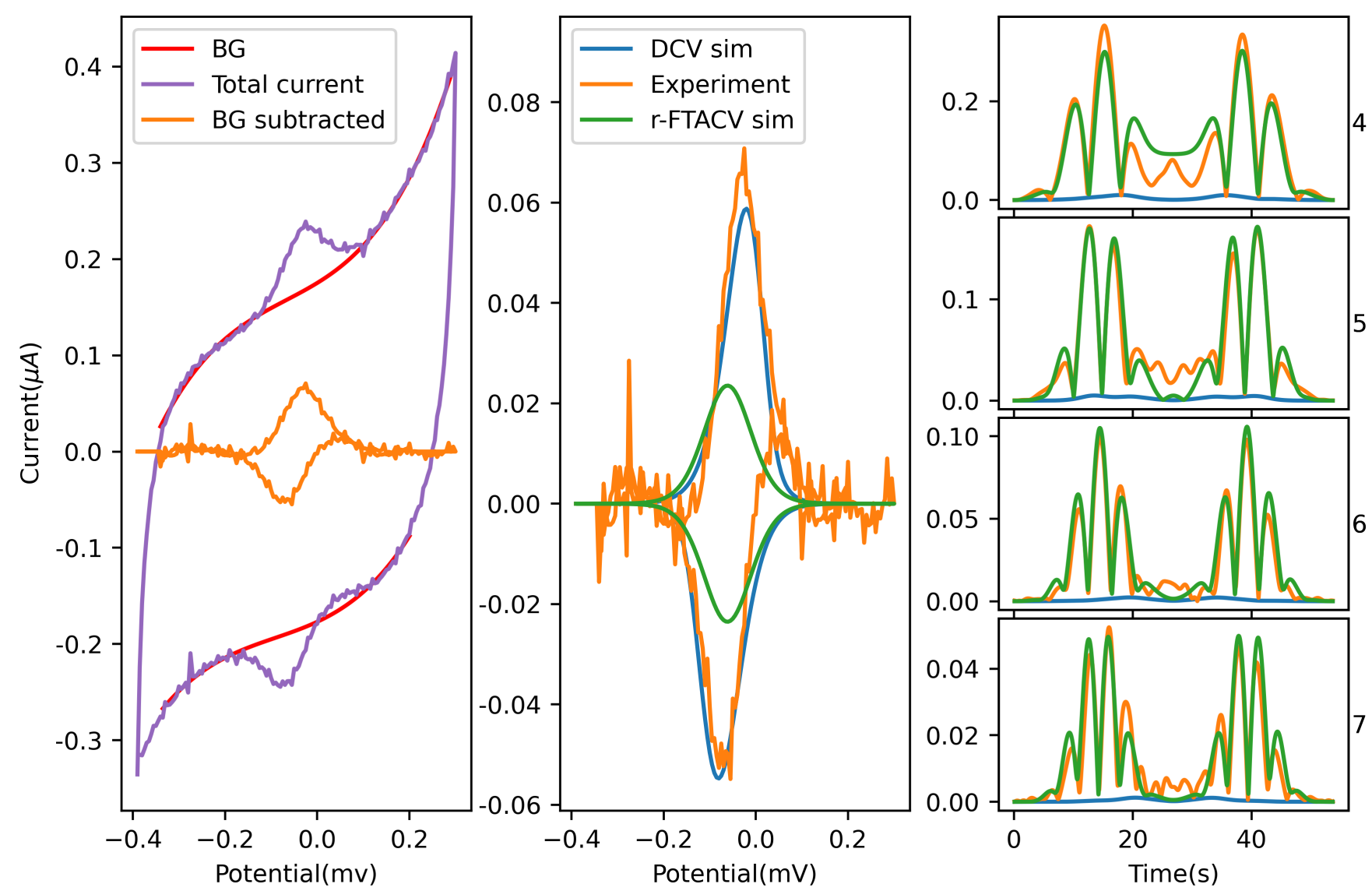

Figure 7: The leftmost plot is an example of the background subtraction procedure which attempts to isolate purely Faradaic current from a DCV experiment. We interpolate across the region of the total DCV current (purple) using a third-order polynomial (red), and subtract this polynomial from the total current to yield the background-subtracted current (orange). In the central plot, we show the same background-subtracted current with two purely Faradaic current simulations. The DCV simulation (blue) was generated using parameters obtained by fitting to the background current, and are recorded in table 3 . The r-FTACV simulation uses parameters listed in table 2 , using the values in brackets where appropriate. In the leftmost plot, the parameters in table 3 fitted to the DCV experiment are used to generate r-FTACV harmonics 4-7, and plotted against r-FTACV data harmonics, alongside the harmonics from the r-FTACV simulation in green.

being a good fit to DCV and a good fit to rFTACV. The poor fit of the DCV parameters to the r-FTACV experimental data is explained by the fact that the inference process predicts a very small kinetic parameter when fitting to the DCV data. The reason for this is because, even though the scan rate is relatively slow, the peak position of the oxidative and reductive peaks is not the same in the background-subtracted current. At the scan rate of this experiment, the peak overlap (i.e. the point of complete kinetic reversibility at this scan rate) occurs at $5 \mathrm{~s}^{-1}$, a value we know to be too low, because even a kinetic value of $20 \mathrm{~s}^{-1}$, as observed in figure 3 . produces r-FTACV harmonics that do not have a shape that corresponds to the experimental data. The non-overlapping oxidative and reductive observed peaks in the backgroundsubtracted DCV current is presumably because the subtraction process does not truly remove all the background current, and we are observing contamination from capacitive current. We do not believe this driven by a problem with the subtraction methodology, as we determined that we could recover the true peak positions using the background-subtraction approach on synthetic data generated by adding simulated Faradaic current to experimental blank data, as 
shown in the Supplementary Information in figure S2. What this tells us is that the observed DCV current is not a simple addition of capacitive and Faradiac current terms (they are convolved, as mentioned in the introduction), and that consequently subtraction is not sufficient to separate the two contributions. Furthermore, any attempt to correct for this observed effect will surely be entirely arbitrary, and we could not have confidence in the inferred values. The poor translation of r-FTACV parameters to the DCV current is presumably driven by differences in surface coverage between the DCV and r-FTACV experiments (as the former was performed before the latter). Our conclusion from this comparison exercise is that our modelling methodology is not well-suited to obtaining information from a single DCV experiment, firstly because our capacitance model struggles to account for the non-idealities observed in actual DCV data, and secondly because the background-subtraction approach does not reduce the problem of capacitance to the same extent as filtering in the Fourier domain does. This conclusion appears to be supported by the literature, where all studies reviewed that attempted to infer kinetic parameters from DCV data used multiple DCV experiments in the form of a trumpet plot, $11+15$ which we discuss below.

\section{Multiple DCV experiments}

Given the challenges associated with analysing a single DCV experiment, we turned to using multiple DCV experiments at different scan rates to generate a so-called "trumpet plot", as pioneered by Laviron. $\frac{34}{34}$ The idea behind this approach is that the scan rate at which the peak potentials diverge is characteristic of the kinetics of the system being interrogated. We found that this approach was less affected by the issues of capacitive current than the singleexperiment approach. In figure 8 we show the trumpet plot data and various fits. Multiple DCV experiments were conducted at different scan rates using the same protein sample as that generated data for PSV and r-FTACV experiment 3 , although using a different electrode
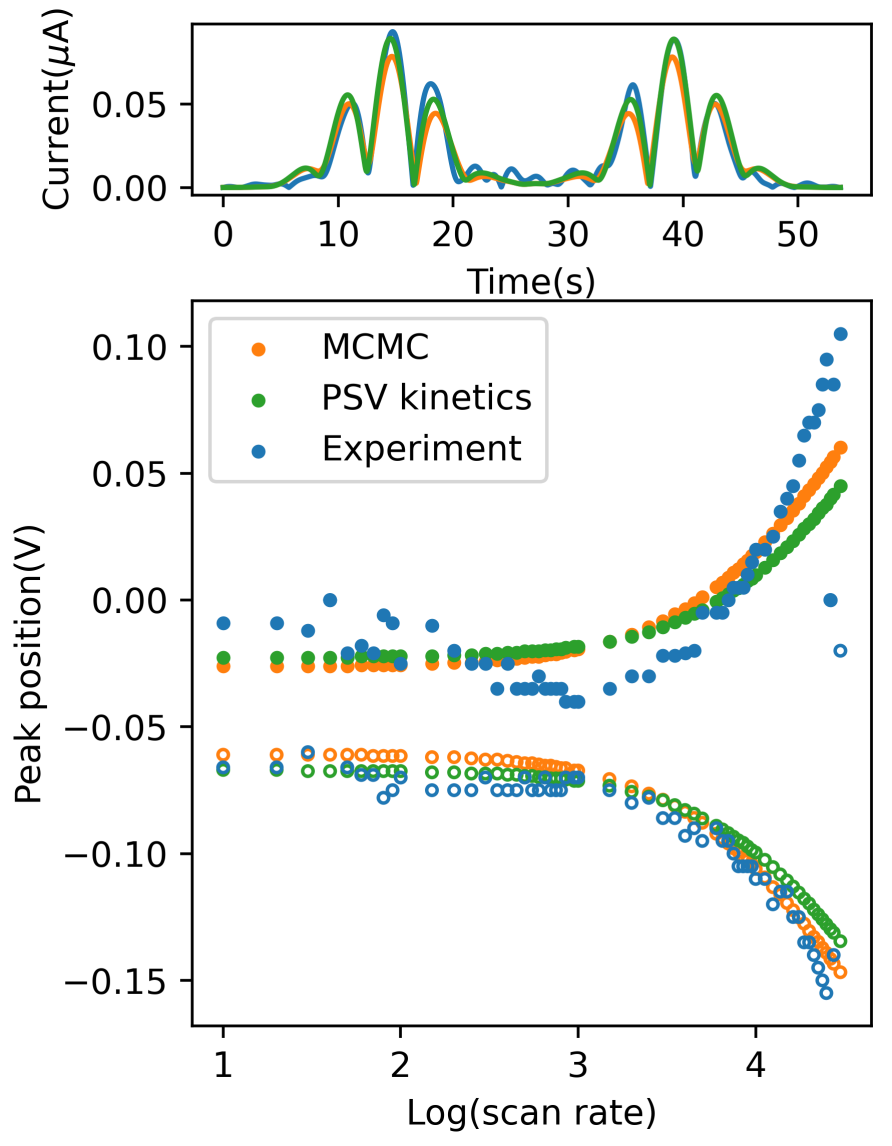

Figure 8: The lower figure shows trumpet plots, plotting the base 10 logarithm of the scan rate against oxidative (filled circles) and reductive (hollow circles) peak positions. Three trumpet simulations are also shown, as reported in table 3. The PSV kinetic simulation differed from the CMAES simulation only by the value of the kinetic parameter, which is written in brackets in table 3 in the appropriate column. The top plot shows harmonic 7 from r-FTACV experiment 3, alongside two simulations. The "PSV kinetics" simulation (red) uses parameters from the PSV 3 column of table2, whereas the "MCMC" simualtion (green) uses the same parameters except for the kinetic value, which is as written in the appropriate column in table 3 
Table 3: Table of inferred DCV parameters. The single DCV column was used to generate the purely Faradaic simulation plotted in the central graph of figure 7 in blue. The parameters for the "trumpet" column were obtained by fitting to the data in figure 8 , where we can only fit $E^{0}$ and $k_{0}$, along with a "separation" parameter that we do not report here, but that is described in the main body of the paper. 2

\begin{tabular}{|c|c|c|c|}
\hline Parameter & Symbol & Single DCV & Trumpet \\
\hline \hline Midpoint potential mean & $E^{0} \mu(\mathrm{V})$ & $-5.100 \mathrm{E}-2$ & \multirow{2}{*}{$-5.928 \mathrm{E}-2$} \\
\cline { 1 - 3 } Midpoint potential s.d. & $E^{0} \sigma(\mathrm{V})$ & $1.000 \mathrm{E}-5$ & \\
\hline Rate constant & $k_{0}\left(s^{-1}\right)$ & 0.587 & \multirow{2}{*}{109.411} \\
\hline Uncompensated resistance & $\mathrm{R}_{u}(\Omega)$ & $3.401 \mathrm{E}-7$ & \multirow{2}{*}{$*$} \\
\cline { 1 - 3 } Surface coverage & $\Gamma\left(\mathrm{mol} \mathrm{cm}^{-2}\right)$ & $3.108 \mathrm{E}-11$ & \\
\hline Symmetry factor & $\alpha$ & 0.452 & \\
\hline
\end{tabular}

film. We also show harmonic 6 from the appropriate r-FTACV experiment, along with the results of simulations generated using parameters in table 3. To generate the trumpet plots, the current was background-subtracted, as shown in 7 , and the position within the potential window of the largest signal of the reductive and oxidative peaks was extracted (from here onwards referred to as "peak position"). A rolling window was used to smooth the backgroundsubtracted experimental data to lessen the impact of experimental noise on the inferred peak position, although this was not successful in all cases, as some outliers away from the general trend can be observed in the trumpet plot of the data in figure 8. To fit this data, we simulated purely Faradaic DCV current at the appropriate scan rate, optimising the value of three parameters. The first was $E^{0}$ without dispersion, in contrast to all previous fitting approaches. This is because the presence of thermodynamic dispersion does not affect the value of the peak position (when taken at the peak maximum). $\underline{31}$ We also fitted $k_{0}$, which controls at what scan rate the peak positions diverge, and a separation parameter. Theory predicts that the oxidative and reductive peak positions must meet at sufficiently slow scan rates, but we did not observe this, presumably because of the same issues observed in analysis of the single DCV experiment discussed above. This artefact was observed in some, $\frac{11 \mid 15}{10}$ but not all, $13 \mid 14$ of the trumpet plot studies cited previously. A constant separation value in volts was added to all oxidative potential positions and subtracted from all reductive potential positions - consequently, we assume that the capacitive artefacts that are unaffected by BG-subtraction are constant, independent of scan rate. We also show the results of two simulations; the first is the mean of the inferred parameter distributions returned by an MCMC process (with the inferred distributions plotted in figure 9, labelled "DCV"). As the reported kinetic value of $\sim 109 \mathrm{~s}^{-1}$ was fairly different from the value inferred from the PSV experiment 3 data (that of $\sim 172 \mathrm{~s}^{-1}$ ), we also checked what the trumpet plot appearance with this kinetic value was, which is the second simulation, labelled "PSV kinetics". It should be noted that some discrepancy is not totally surprising, as the trumpetplot data was collected from a different electrode film from PSV experiment 3, although both films were generated from the same protein extract. The values of these parameters are recorded in table 3. In terms of the actual experimental data, the gradient of the oxidative and reductive peak potentials is different, and there is significant deviation from the the predicted largely straight line for the oxidative peak position between the $\log$ (scan rate) values of 1-3. Although there is a difference between the kinetic values inferred using DCV and PSV $\left(\sim 60 \mathrm{~s}^{-1}\right)$, this difference does not significantly change the observed fits. In figure 8, we show a trumpet plot obtained using a kinetic value of $173 \mathrm{~s}^{-1}$ (labelled "PSV kinetics") and a ramped simulation generated using the kinetic value in- 
ferred from DCV; in both cases, the increase or decrease of $\sim 60 \mathrm{~s}^{-1}$ did not significantly affect the goodness of fit to the experimental current. The kinetic values inferred from the various experiments are very much in the same regime, in contrast to, for example figure 3, where a large difference in the kinetic value used to generate two simulations results in a totally different harmonic appearance. Our conclusion from this analysis was that the trumpet-plot method is significantly more useful than analysis of a single DCV experiment, and can provide validation of the kinetics of PSV-inferred parameter vectors, along with information for how best to bound the kinetic parameter space.

\section{Confidence and uncertainty}

All results and consequent discussion up to this point have been obtained using the CMAES algorithm, and consequently the parameter values discussed above are inferred point values. However, we can also use MCMC methods to obtain a description of the amount of confidence we have in our model. This is shown in figure 9. For each MCMC simulation, 3 Markov chains were run independently, starting from the parameters reported in table 2 , run for 10000 iterations and then combined, again fitting to the 4th harmonic and above from the PSV experiment. The DCV parameters were as inferred from the trumpet plot, and as such we only report the inferred $E^{0}$ (on the same graph as the PSV $E^{0} \mu$ values, as the parameters are comparable), and $k_{0}$ values. Because of issues associated with the MCMC algorithm and bounding, the $C_{d l}$ and $\alpha$ values were not included in the inference process, and instead set at the value detailed in table 2. The traces for each MCMC process used to calculate the plotted histograms can be found in the supplementary information, in figures S3-6 The inferred PSV parameter distributions shown are very narrow, implying a high degree of confidence in the parameters listed in table 3 , in contrast to the much broader inferred DCV parameter distributions.

It is interesting that this approach cannot cap- ture the magnitude of the parameter compensation phenomenon we discussed with regards to the inferred point values above. As discussed, the parameters reported were drawn from a subsection of parameter space that generated uniformly good fits. As we did not include the $C_{d l}$ and $\alpha$ parameters in the inference process, we are effectively exploring a sub-section of parameter space where these values are fixed, which accounts for the very high level of confidence observed. If in future work we can resolve these issues, we predict we will see somewhat broader inferred parameter distributions. Therefore these results should be treated with some caution, and mainly show the difference between the width of the distributions for the PSV and DCV cases. Another utility of the MCMC approach is shown in figure 10, where we plot all MCMC inferred parameter distributions for the inference process linked to PSV experiment 2, allowing us to see correlations between parameters. As shown in the figure, there is a high degree of pairwise correlation, with some particularly strong examples of parameter correlation being between $k_{0}$ and $\eta$, and $\Gamma$ and $E^{0} \sigma$.

\section{Conclusions}

In this paper we have shown that it is possible to obtain a highly detailed picture of the electrochemistry of the single-electron redox reaction of Cjx183, to the extent that the parameters we have obtained are consistent across multiple experiments using different preparations of the same enzyme. We have shown how the multi-experiment approach, coupled with Bayesian inference, allows for increased confidence that the parameters we report are in a regime that is a good reflection of the underlying chemistry. For future work, we intend to extend our repertoire of techniques to include square wave voltammetry and electrochemical impedance spectroscopy, and to move towards systems that have more complex chemistries, including multiple electron-transfer reactions and catalytic processes. For researchers looking to use this methodology, we propose the follow- 

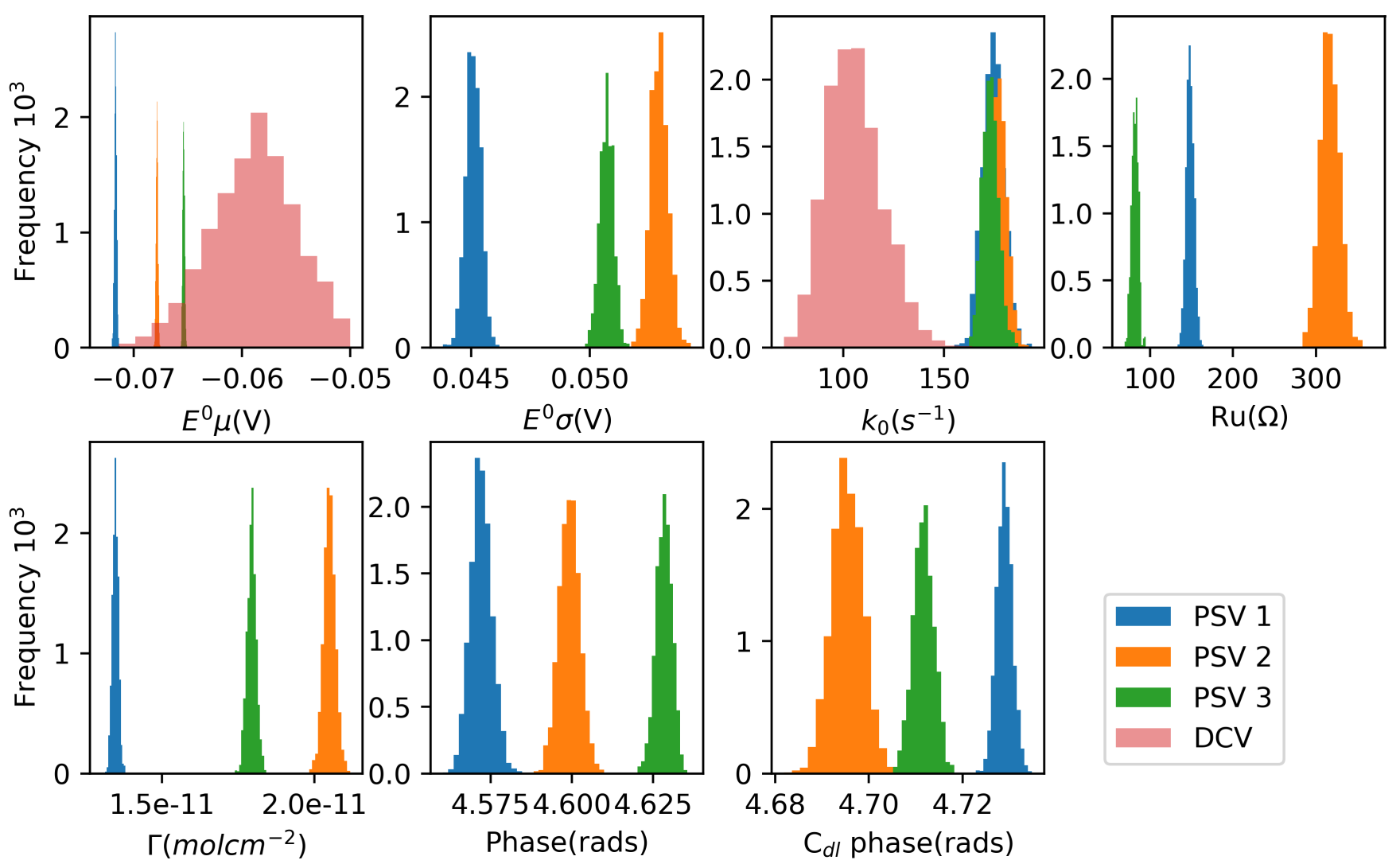

$\operatorname{Ru}(\Omega)$

Figure 9: Inferred parameter histograms generated by pooling three independent MCMC chains, using harmonics 4 and above of the respective PSV experiments in the likelihood function, and using the parameters in table 2 as the starting point. $C_{d l}$ and $\alpha$ were not included in this parameter inference approach for technical reasons mentioned in the text. The DCV histograms were as inferred from running an MCMC process on the trumpet data in figure 8 , and as such we can only infer the parameters $E^{0}$ and $k_{0}$

ing "recipe" for robustly inferring parameters.

1. Collect experimental data. For each electrode functionalised with protein, we recommend collecting PSV, r-FTACV and DCV data (the latter at different scan rates), in that order. This is because there will naturally be some film-loss as a result of consecutive experiments, and consequently we order the experiments in order of how important having good signals is.

2. Obtain estimates for $E^{0}$ and $k_{0}$ bounds by fitting trumpet plot data, which is relatively easy to fit. This information should then be used to judge parameters inferred from analysis of PSV and r-FTACV data. You can also use MCMC at this juncture to explore the confidence in the inferred values.

3. Define boundaries. Initial boundaries should be encompass a reasonably large area of parameter space, but as a rule of thumb should not cover more than two orders of magnitude. If this coverage is necessary, consider log-transformations.

4. Determine if it is feasible to fit PSV data in the time domain, without using dispersion. If inferred Faradaic parameters are highly divergent between different fitting runs, then you need to fit in the Fourier domain. If this is the case, inspect the harmonics of the blank PSV data to see what portions of the Fourier domain needs to be zeroed-out 


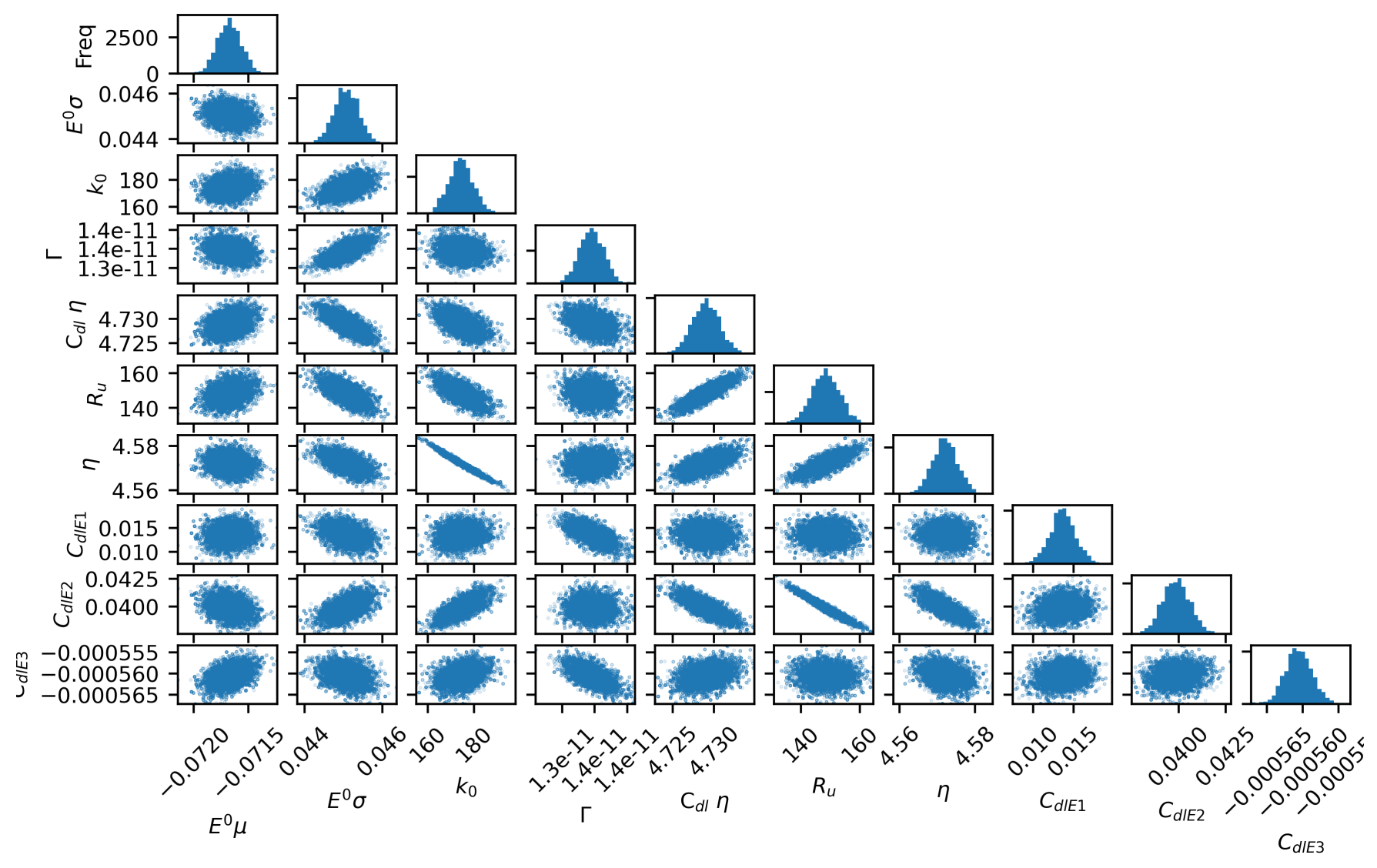

Figure 10: 2D histograms generated from the MCMC process for PSV experiment 2

5. Fit the form of the PSV data chosen above, using a simulation without dispersion. Using the parameters resulting from this inference process, generate a ramped simulation and compare to the r-FTACV data harmonics to assess the translatablity of the parameters. It should be reasonably clear if you are neglecting thermodynamic dispersion, as the simulated harmonics will be narrower, and will not decrease in magnitude with harmonic number to the extent observed in the experimental data. If thermodynamic dispersion is present, you should go back to fitting the PSV data accordingly. If the kinetics of the system are irreversible/quasi-reversible then it may be worth considering kinetic dispersion as well, as discussed in previous work,, 31 but we have not encountered this scenario to date.

6. Keep on comparing your PSV fits to the r-FTACV harmonics. Some other points to note:

- If fitting to the Fourier domain, keep checking that the predicted total current simulation is not significantly greater in magnitude than the experimental current data, an issue we came against in figure 4.

- If a parameter is consistently hitting a defined boundary, then consider raising or lowering this bound as appropriate, unless this is outside of the realms of chemical plausibility. Beware of parameter compensation effects!

- A good rule of thumb is that you will see a set of "good-fit parameters" at least once in ten fitting runs with a random initialisation.

7. Choosing which parameters to report is something of a personal choice - our rationale was that the inferred parameters for the three experiments should be in the 
same regime while providing a good fit to each.

\section{References}

(1) Beaton, S. E.; Evans, R. M.; Finney, A. J.; Lamont, C. M.; Armstrong, F. A.; Sargent, F.; Carr, S. B. The structure of hydrogenase-2 from Escherichia coli: implications for H2-driven proton pumping. Biochemical Journal 2018, 475, 13531370 .

(2) Fourmond, V.; Wiedner, E. S.; Shaw, W. J.; Léger, C. Understanding and design of bidirectional and reversible catalysts of multielectron, multistep reactions. Journal of the American Chemical Society 2019, 141, 11269-11285.

(3) Lloyd-Laney, H. O.; Yates, N. D.; Robinson, M. J.; Hewson, A. R.; Firth, J. D.; Elton, D. M.; Zhang, J.; Bond, A. M.; Parkin, A.; Gavaghan, D. J. Using Purely Sinusoidal Voltammetry for Rapid Inference of Surface-Confined Electrochemical Reaction Parameters. Analytical Chemistry 2021, 93, 2062-2071.

(4) Bond, A. M.; Duffy, N. W.; Guo, S.-X.; Zhang, J.; Elton, D. Changing the look of voltammetry. Analytical chemistry 2005, 77, 186-A.

(5) Adamson, H.; Bond, A. M.; Parkin, A. Probing biological redox chemistry with large amplitude Fourier transformed ac voltammetry. Chemical Communications 2017, 53, 9519-9533.

(6) Yates, N. D.; Dowsett, M. R.; Bentley, P.; Dickenson-Fogg, J. A.; Pratt, A.; Blanford, C. F.; Fascione, M. A.; Parkin, A. Aldehyde-mediated proteinto-surface tethering via controlled diazonium electrode functionalization using protected hydroxylamines. Langmuir 2019, 36, 5654-5664.
(7) Gavaghan, D. J.; Cooper, J.; Daly, A. C.; Gill, C.; Gillow, K.; Robinson, M.; Simonov, A. N.; Zhang, J.; Bond, A. M. Use of Bayesian inference for parameter recovery in DC and AC Voltammetry. ChemElectroChem 2018, 5, 917-935.

(8) Bieniasz, L.; Speiser, B. Use of sensitivity analysis methods in the modelling of electrochemical transients: Part 3. Statistical error/uncertainty propagation in simulation and in nonlinear least-squares parameter estimation. Journal of Electroanalytical Chemistry 1998, 458, 209-229.

(9) Gulaboski, R.; Mirčeski, V.; Bogeski, I.; Hoth, M. Protein film voltammetry: electrochemical enzymatic spectroscopy. A review on recent progress. Journal of Solid State Electrochemistry 2012, 16, 23152328.

(10) Léger, C.; Bertrand, P. Direct electrochemistry of redox enzymes as a tool for mechanistic studies. Chemical Reviews 2008, 108, 2379-2438.

(11) Stan, R. C.; Kros, A.; Akkilic, N.; Sanghamitra, N. J.; Appel, J. Direct wiring of the azurin redox center to gold electrodes investigated by protein film voltammetry. Journal of Electroanalytical Chemistry 2017, 787, 14-18.

(12) Fleming, B. D.; Zhang, J.; Elton, D.; Bond, A. M. Detailed analysis of the electron-transfer properties of azurin adsorbed on graphite electrodes using dc and large-amplitude Fourier transformed ac voltammetry. Analytical chemistry 2007, 79, 6515-6526.

(13) Armstrong, F. A.; Heering, H. A.; Hirst, J. Reaction of complex metalloproteins studied by protein-film voltammetry. Chemical Society Reviews 1997, 26, 169-179.

(14) Armstrong, F. A. Insights from protein film voltammetry into mechanisms of complex biological electron-transfer reactions. Journal of the Chemical Society, Dalton Transactions 2002, 661-671. 
(15) Hirst, J.; Armstrong, F. A. Fast-scan cyclic voltammetry of protein films on pyrolytic graphite edge electrodes: characteristics of electron exchange. Analytical chemistry 1998, 70, 5062-5071.

(16) Munge, B.; Das, S. K.; Ilagan, R.; Pendon, Z.; Yang, J.; Frank, H. A.; Rusling, J. F. Electron transfer reactions of redox cofactors in spinach photosystem I reaction center protein in lipid films on electrodes. Journal of the American Chemical Society 2003, 125, 1245712463.

(17) Jeuken, L. J.; McEvoy, J. P.; Armstrong, F. A. Insights into gated electrontransfer kinetics at the electrode- protein interface: a square wave voltammetry study of the blue copper protein azurin. The Journal of Physical Chemistry B 2002, 106, 2304-2313.

(18) Huang, X.; Wang, L.; Liao, S. Method of evaluation of electron transfer kinetics of a surface-confined redox system by means of Fourier transformed square wave voltammetry. Analytical chemistry 2008, $80,5666-5670$.

(19) Lee, C.-Y.; Fleming, B. D.; Zhang, J.; Guo, S.-X.; Elton, D. M.; Bond, A. M. Systematic evaluation of electrode kinetics and impact of surface heterogeneity for surface-confined proteins using analysis of harmonic components available in sinusoidal large-amplitude Fourier transformed ac voltammetry. Analytica chimica acta 2009, 652, 205-214.

(20) Adamson, H.; Robinson, M.; Wright, J. J.; Flanagan, L. A.; Walton, J.; Elton, D.; Gavaghan, D. J.; Bond, A. M.; Roessler, M. M.; Parkin, A. Retuning the catalytic bias and overpotential of a [NiFe]-hydrogenase via a single amino acid exchange at the electron entry/exit site. Journal of the American Chemical Society 2017, 139, 10677-10686.
(21) Gulaboski, R.; Mirceski, V.; Lovric, M. Square-wave protein-film voltammetry: new insights in the enzymatic electrode processes coupled with chemical reactions. Journal of Solid State Electrochemistry 2019, 23, 2493-2506.

(22) Hexter, S. V.; Esterle, T. F.; Armstrong, F. A. A unified model for surface electrocatalysis based on observations with enzymes. Physical Chemistry Chemical Physics 2014, 16, 11822-11833.

(23) Fourmond, V.; Léger, C. Modelling the voltammetry of adsorbed enzymes and molecular catalysts. Current Opinion in Electrochemistry 2017, 1, 110-120.

(24) Hirst, J. Elucidating the mechanisms of coupled electron transfer and catalytic reactions by protein film voltammetry. Biochimica et Biophysica Acta (BBA)Bioenergetics 2006, 175\%, 225-239.

(25) Léger, C.; Jones, A. K.; Albracht, S. P.; Armstrong, F. A. Effect of a dispersion of interfacial electron transfer rates on steady state catalytic electron transport in [NiFe]-hydrogenase and other enzymes. The Journal of Physical Chemistry B 2002, 106, 13058-13063.

(26) Merrouch, M.; Hadj-Saïd, J.; Léger, C.; Dementin, S.; Fourmond, V. Reliable estimation of the kinetic parameters of redox enzymes by taking into account mass transport towards rotating electrodes in protein film voltammetry experiments. Electrochimica Acta 2017, 245, 10591064.

(27) Reda, T.; Hirst, J. Interpreting the catalytic voltammetry of an adsorbed enzyme by considering substrate mass transfer, enzyme turnover, and interfacial electron transport. The Journal of Physical Chemistry B 2006, 110, 1394-1404.

(28) Branch, J.; Rajagopal, B. S.; Paradisi, A.; Yates, N.; Lindley, P. J.; Smith, J.; Hollingsworth, K.; Turnbull, W. B.; 
Henrissat, B.; Parkin, A.; Berry, A.; Hemsworth, G. R. unpublished.

(29) Leggio, L. L.; Simmons, T. J.; Poulsen, J.-C. N.; Frandsen, K. E.; Hemsworth, G. R.; Stringer, M. A.; Von Freiesleben, P.; Tovborg, M.; Johansen, K. S.; De Maria, L., et al. Structure and boosting activity of a starch-degrading lytic polysaccharide monooxygenase. Nature communications 2015, 6, 1-9.

(30) Branch, J. Activation of a bacterial Lytic Polysaccharide Monooxygenase using a small c- type cytochrome. Submitted to Journal of Biological Chemistry 2021,

(31) Lloyd-Laney, H. O.; Robinson, M. J.; Bond, A. M.; Parkin, A.; Gavaghan, D. J. A Spotter's Guide to Dispersion in NonCatalytic Surface-Confined Voltammetry Experiments. Journal of Electroanalytical Chemistry 2021, 115204.

(32) Adamson, H.; Robinson, M.; Bond, P. S.; Soboh, B.; Gillow, K.; Simonov, A. N.; Elton, D. M.; Bond, A. M.; Sawers, R. G.; Gavaghan, D. J., et al. Analysis of HypD disulfide redox chemistry via optimization of fourier transformed ac voltammetric data. Analytical chemistry 2017, 89, 1565-1573.

(33) Feldberg, S. W. Effect of uncompensated resistance on the cyclic voltammetric response of an electrochemically reversible surface-attached redox couple: Uniform current and potential across the electrode surface. Journal of Electroanalytical Chemistry 2008, 624, 45-51.

(34) Laviron, E. General expression of the linear potential sweep voltammogram in the case of diffusionless electrochemical systems. Journal of Electroanalytical Chemistry and Interfacial Electrochemistry 1979, 101, 19-28. 